\title{
The Geometry of Environmental knowledge
}

\author{
Daniel R. Montello \\ Department of Geography, University of California \\ Santa Barbara, CA 93106-4060 \\ USA
}

\begin{abstract}
Theoretical and empirical work on the geometry of environmental knowledge is discussed. Certain patterns of distance and directional estimates collected from humans have been interpreted as being due to non-metric or non-Euclidean spatial knowledge. I argue that attempts to determine this geometry are inconsistent with existing theoretical models of human knowledge storage and use. spatial estimation data are more parsimoniously explained as resulting from: (a) measurement error and ambiguity, (b) multiple and uncoordinated long-term memory representations, and (c) processes taking place when spatial knowledge is activated and used.
\end{abstract}

\section{Introduction}

The philosopher Kant maintained that phenomenal space such as that experienced during visual perception or imagery is described by Euclidean geometry [37, 42]. since space is readily perceived and imagined in this way, Kant reasoned, physical space must be Euclidean. In Kant's terms, the 3-dimensional Euclidean structure of space is synthetic (nontrivial, meaningful description of the world) and a priori (self-evident and not subject to empirical test). This view is intuitively appealing, as for instance when a 3-dimensional Euclidean sphere is provided for the "visualization" of 2-dimensional Riemannian space. But Kant's arguments for the selfevident geometry of physical space have been criticized frequently, and it is now believed that Euclidean geometry does not best describe the space of the physical universe (e.g., Einstein's general theory of relativity). 
But what about the space of the mind -- "space" as it is perceived, imagined, and stored in memory? The intuitive appeal of Euclidean geometry may imply something about psychological space that it does not imply about physical space. The fact that space at scales of size relevant to certain topics in physics and astronomy may best be described as non-Euclidean (or hyperdimensional, etc.) does not force us to adopt such models for space at scales relevant to psychology:

"The description of reality with the help of the four-dimensional schema [one of time] is a result of the construction of physical space from psychological spaces (visual and tactile spaces, etc.). These latter are, however, by no means relative... In these regions, the application of Euclidean geometry is more than an arbitrary convention. Nevertheless, the question as to which geometries shall be constructed for greater or smaller magnitudes, that is, for atomic or astronomical dimensions, is no longer psychologically, but purely physically, determined". [38, p. 296]

In the following essay, I discuss some recent theoretical and empirical work on the geometry of environmental knowledge. Properties of metric spaces in general and Euclidean metric spaces in particular are briefly reviewed. I present a representative sample of research on the estimation of spatial properties of the environment (distance and directional relationships) that has implications for the geometry of environmental knowledge. The importance of several measurement issues is described. Finally, I question assertions about the geometry of environmental knowledge, primarily with respect to implicit assumptions about memory structure and the processes required to externalize this structure as thought and behavior.

My discussion below focuses on knowledge of largescale or environmental spaces, spaces that surround us and generally require movement over time for their apprehension. Because these spaces apparently necessitate considerable information integration in memory over time, their mental representations have been termed cognitive maps or cognitive spaces $[6,8,10,18]$. In contrast, perceptual spaces are those apprehended relatively simultaneously during perception, usually 
visual. Evidence in fact suggests that the perspective geometry of perceptual, or visual, space may not best be described as Euclidean. The Luneberg-Blank theory states that visual space follows a homogeneous, hyperbolic metric $[4,11,17,26]$; straight lines behave as though they were on the surface of a hyperbolic plane. Not all features of this theory are universally accepted (Foley [11], for instance, questions the homogeneity of visual space), but the evidence is clearly at odds with a Euclidean metric.

Acknowledging the non-Euclidean nature of perceptual space, however, does not force us to adopt such a geometry for internal spatial representations derived from perceptual events. Encoding processes operating on perceptual input could transform hyperbolic space into Euclidean space, or for that matter, into some other nonEuclidean or non-metric space. For instance, Baird, Wagner, and Noma [3] propose that such processes transform the locally Euclidean metric of the environment into non-Euclidean cognitive space.

\section{Alternative Geometries in the study of Environmental Knowledge}

Euclidean space is one member of a general class of metric spaces, spaces for which quantitative distances between locations can be defined in a consistent way that does not depend on the direction of measurement between two locations. Metric spaces are characterized by the following axioms (d is distance; $m, n$, and o are locations in space):
a) $d(m, n) \geq 0$
b) $d(m, n)=0$,
if and only if $m$ and $n$ coincide
c) $d(m, n)=d(n, m)$
nonnegativity
nondegeneracy
d) $d(m, n)+d(n, 0) \geq d(m, 0)$
symmetry

Metric spaces include, but axe not limited to, Euclidean spaces. Two-dimensional Euclidean spaces are the flat, infinite, continuous, and isotropic spaces familiar from high school geometry and formally characterized by the five postulates of Euclid's geometry [14, 37].

Euclid's fifth postulate has been the most controversial philosophically. In one of its forms, it states: 
Given a straight line and a point not on that line, there is one and only one straight line through the point and parallel to the original line.

Various non-Euclidean metric geometries, such as hyperbolic and spherical geometries, take issue with the fifth postulate, curving planar space so that no parallels or many parallels exist. Alternatively, nonEuclidean metric spaces may be flat but locally nonisotropic. Straight-line distance in these metric but non-Euclidean spaces depends in part on the angular orientation of the points in the space [13,40]. The city-block metric, for instance, is a flat metric that requires distance measurement along orthogonal lines that connect the endpoints, much as road distances in cities follow city blocks.

In the psychological literature on cognitive distance, the term Euclidean distance has often been equated with straight-line or as-the-crow-flies distance, in contrast to route or functional distance [e.g., 35, 44]. This usage is inexact, however, given that the shortest route between locations on a 2-dimensional surface (the "straight-line" route) would not be a Euclidean straight line if the space was non-Euclidean. An example is the straight-line route of an airplane flying from New York to Moscow -- it follows a great circle, or circumference line, around the globe. Conversely, route distance may or may not be based on a representation with the properties of a Euclidean space, though it would equal straight-line distance only along routes without turns.

The most important historical example of explicit theorizing about the geometry of psychological, as opposed to physical, space comes from the extensive work of Piaget [cf. 33]. He and his colleagues proposed that spatial knowledge develops ontogenetically from topological to projective and fully Euclidean geometry in the mature thinker. However, he did not explicitly attempt to empirically evaluate the geometry of the adult's spatial knowledge in a rigorous fashion.

More recently, psychologists, geographers, and others have attempted to empirically evaluate the metric of environmental knowledge. There are several strategies for doing this. One is to examine estimates of distances in the environment: Are they consistent with the metric axioms? In fact, several researchers have reported 
violations of the symmetry and triangle inequality axioms $[3,7,29,31,36,43]$. Sadalla et al. [36], for example, found that distance from a campus building high in reference-point saliency to a campus building low in reference-point saliency was estimated as being greater than the same distance in the opposite direction. They attributed this to the role of reference points in the organization of environmental memory representations.

A strategy for determining which metric best describes the geometry of spatial knowledge follows from a theorem derived from Euclid's fifth postulate about parallels; The sum of the angles of the vertices of a triangle will equal $180^{\circ}$ if the space is Euclidean, within the limits of measurement error. In the 19th century, Gauss actually attempted to establish the geometry of physical space by summing the angles formed by light rays traveling between three distant mountain peaks ([42) -Gauss concluded that within limits of measurement accuracy, physical space was Euclidean). By a similar logic, the sum of the vertex angles of a triangle formed by the estimation from memory of the directions between three locations should equal $180^{\circ}$ if the knowledge space is Euclidean. For example, Moar and Bower [29] found that the sum of estimated directions between three nonvisible street intersections significantly exceeded $180^{\circ}$ in several cases. Although they did not interpret it as such, this finding is consistent with a spherical geometry of strong curvature.

Perhaps a more sophisticated approach to this question has been the use of multidimensional scaling (MDS) algorithms to fit a geometry to a matrix of proximity estimates made between locations. Solutions obtained using spaces of different metrics are compared with respect to their stress values, their abilities to reproduce the original values of the proximity estimates [e.g., 13, 45]. The relative stress of Euclidean and non-Euclidean solutions $c a n$ be compared. Golledge and Hubert [13], for instance, report data from an earlier study of distance estimation within the city of columbus. The distance estimates of $18 \%$ of residents were better fit by a non-Euclidean metric than they were by a Euclidean metric. However, these authors go on to discuss research by spector and by Richardson in which matrices of distance estimates at intraurban scales were not fit as well by a Euclidean as by a non-Euclidean 
metric (particularly the city-block metric).

Shepard [40] discusses the MDS approach and some difficulties associated with it, particularly when comparing a Euclidean metric to alternative flat but nonisotropic metrics such as the city-block. Because of differences in the degree of degeneracy of the solutions obtained with these different metrics, expected stress values under random conditions are not equal for the different metrics. Certain extreme metrics will artifactually produce low-stress solutions. Furthermore, if nonmetric paired-comparison data are used as input in the MDS program, the various metrics differ very little in their rank ordering of pairwise distances (the Euclidean and city-block, for instance, would generally differ by no more than one pair reversal). Shepard concludes that "the generally accepted practice of taking, as the correct metric, the one which yields the lowest residual departure from monotonicity is unfounded and probably leads to erroneous conclusions" ( $p$. 401).

Although shepard's [40] comments are relevant, a couple of caveats about them should be made. The first is that the difficulties of stress comparisons with different metrics may not be as serious when one starts with the objective spatial configuration (i.e., the actual layout of the urban area being estimated) as a starting point for the iterative fitting done by the MDS algorithm. In addition, different metrics do not reproduce rank orders of pairwise estimates as equivalently when one uses incomplete pairwise designs (as has typically been the case) instead of all possible pairwise estimates. Both issues call for further research ${ }^{1}$.

\section{Measurement and Methodology}

As long as any arbitrary pattern or amount of error can be attributed to the measurement process, any geometry for environmental knowledge can be maintained in the face of any empirical evidence [see 3, 5, 12]. The mathematician Poincaré pointed out some time ago that the appropriateness of a Euclidean metric can only be empirically tested if one makes certain coordinating definitions $[14,37]$. He maintained that decisions about the geometry of a space are conventional, not empirical, because any pattern of data could be explained either by 
characteristics of the space or by characteristics of the behavior of the measuring device as it is moved about the space [42]. Thus, the requirement that one's measuring rod must remain a constant length across measurements is an important historical example of a coordinating definition.

In a recent article [30], I discuss several issues that are relevant to a coordinating definition about the psychophysical measurement of cognitive distance. One is that subjects generally may have different internal representations of standard distances (the mental "yardsticks"), differences which are generally opaque to the researcher. Another is that magnitudes of estimates made with traditional psychophysical methods such as ratio- and magnitude-estimation apparently depend in systematic ways on the relative sizes of the standard distances used in the estimation procedure. To the degree that such factors influence the magnitudes of estimated distances, even estimates derived from Euclidean representations might be interpreted as indicating non-Euclidean or non-metric geometries.

Failures of the metric axioms in the context of cognitive distance measurement were cited above. In general, however, failures of the metric axioms are ubiquitous whenever pairwise estimates of similarity or proximity are collected [46]. Given stimuli that vary along qualitatively different dimensions (an apple has size, color, aroma, etc.), shifts of context and attention will result in violations of the metric axioms [39]. Users of MDS usually transform or otherwise modify their data so it can be fit with a Euclidean or some other metric representation, preventing the description of the resulting spaces as being non-metric.

Furthermore, people apparently do not always estimate distances along Euclidean straight lines even when their representations would allow them to do so. For example, a city-block metric most accurately describes spaces in which shortest distances between places are along routes containing orthogonal turns instead of along straight routes. But just because one knows that the shortest routes that can actually be traveled are not straight lines does not mean that one's mental representation of the space containing the routes is necessarily nonEuclidean. Maps generated from people's distance estimates may be best fit by a city-block metric [13]; 
this may just reveal that unless carefully specified, people will tend to estimate distances along accessible routes.

Data collected by Rieser et al. [35] provide some support for this contention. When given no explicit instructions either to estimate route or straight-line distances, their subjects tended to estimate distances as if they had received route instructions. However, when given straight-line instructions, they estimated distances differently -- more like the actual relative straight-line distances. Thus, subjects were not necessarily bound to a city-block metric. They did tend to interpret the estimation task and use their representations differently under different instructions.

Finally, some additional methods that have been used to measure cognitive distance do not allow tests of the geometry of environmental knowledge. Methods that involve the construction of a spatial representation by simultaneous estimation of locations typically force a Euclidean metric onto the representation (or whatever space we take as a description of flat pieces of paper). Examples are map-drawing and model-building tasks.

\section{Environmental knowledge: structures and Processes}

Given that environmental knowledge must be finite (as are all brains and information storage devices), the question becomes whether it can best be described as locally Euclidean. Behavioral data, including sketch maps, distance estimates, proximity rankings, and directional judgments, indicate that knowledge of the environment is incomplete, distorted, asymmetric, discontinuous, and imperfectly coordinated [e.g., 7, 15, 25, 27, 29]. A uniform or homogeneous metric would not typically describe it well. People store very different amounts of information about different places, often excluding unfamiliar areas altogether. Discrete, fragmented pieces of represented environments are stored at multiple hierarchical levels [28].

Several theorists $[9,22]$ have criticized the concept of cognitive maps as falsely implying that environmental knowledge exists in a unitary spatial format, as in a cartographic map. They instead propose modeling environmental knowledge as a conglomeration of multiple 
representations of space in ITM that are not necessarily coordinated nor mutually consistent, at least not directly. Some of it is not even locally metric, but encodes topological information in non-spatial formats such as verbal and abstract propositional networks (cf. a model by Kuipers and levitt [23] for navigation by autonomous vehicles). From this perspective, environmental long-term memory (ITM) is clearly not a unitary metric representation that is uniform and continuous. Instead, these models suggest that there isn't a aingle psychological space stored in LTM but rather several spaces -- spaces whose LTM characteristics may not even be spatial.

Given this, using sets of distance or directional estimates to conclude that the space of environmental knowledge is non-Euclidean or even non-metric [e.g., 13, 45] might be considered inappropriate. There is no reason that environmental spatial knowledge should fit any single metric unless it comes from a unitary representation. For example, one might estimate the distance from $A$ to $B$ using a different representation than that used when estimating the distance from $B$ to $A$, resulting in asymmetric estimates [cf. 36]. As long as the asymmetric estimates are not simultaneously experienced by the estimator, it is possible for the two mutually inconsistent but internally metric representations to coexist in LTM without conflict. (Moar and Bower [29] provide a similar interpretation of apparently non-Euclidean directional estimates). Such an example illustrates the way that statements about the geometry of environmental knowledge are like constructing a patchwork made up of pieces cut from several maps of different styles and sizes, and then remarking on the distorted, non-metric quality of the resulting patchwork.

But there is another serious difficulty with using data from spatial estimation tasks to model the geometry of environmental knowledge. It involves the processes required to activate LTM spatial knowledge and construct spaces in working memory (WM) during the estimation task. such processes are also required during many everyday uses of environmental knowledge, such as non-automatized wayfinding and direction-giving.

Holyoak and $\operatorname{Mah}$ [16] discuss transformations in spatial knowledge induced by the retrieval of LTM knowledge during distance estimation tasks. Their 
implicit scaling model states that information from LTM is retrieved when estimating distances and used to construct a "scaled" representation of the space in WM. These representations are categorically structured internal scales of distance from which distance estimates can be made. The reference point used to construct the distance scales will affect the way distance is partitioned on the scale (spatial estimates are relative in so far as they require implicitly generated or explicitly provided points of reference). The discrimination categories make up an interval scale in which categories nearest the reference point will contain the least distance. Greater discriminability of distance is thus found near the reference point. As examples, Holyoak and Mah showed that cities in the western US were estimated as being further apart relative to the Pacific ocean than to the Atlantic Ocean. They also used their implicit scaling model to explain patterns of asymmetric distance estimates associated with reference points [36], favoring such an explanation in this case over a model that posits multiple ITM representations for estimates in opposite directions (as I discussed above).

Presumably such a scaling process has evolved as the most efficient way to access spatial information, given limitations on how much information can be maintained in WM (it is useful to be able to make finer discriminations near points of reference). Alternatively, the greater density of knowledge near personal reference points (such as one's home) will lead to a greater representation of information near the reference point on the WM scale. Either way, implicit scaling results in the "stretching" of subjective distance in the vicinity of reference points. For purposes of the present discussion, the distortion from geographic reality resulting from such scaling processes in a single represented space is not so important (it might still result in a Euclidean space). More importantly, such processes operating differently over the course of data collection would typically produce mutually non-metric estimates. Thus, a particular knowledge structure in LTM will be activated and used in WM differently depending on the nature of the task at hand. "Psychological spaces don't exist sui generis; they are constructed on the spot, and their nature and field of view will be task specific" [19, pp. 23-24]. 


\section{Conclusions}

Both the pluralistic nature of ITM spatial knowledge and knowledge activation theories such as the implicit scaling model [16] provide reasons that spatial estimation data would not be fit well by a Euclidean metric (or perhaps any metric). These ideas in fact suggest that such an enterprise is mistaken, in so far as it involves the implicit or explicit assumption that environmental knowledge can be meaningfully modeled as a coordinated, unitary representation. Such attempts typically fail to take account of the distinction between WM and ITM knowledge. "Considerable confusion has been engendered by a failure to distinguish between spatial thought [WM] and spatial storage [LTM]" [24, p. 13]. Because behavioral data (maps, distance or proximity estimates, directional judgments) are usually derived from multiple representations that are not simultaneously activated into an internally consistent wM representation, it should come as no surprise that the resulting data violate metric axioms. This does not imply that cognitive space is non-metric or nonEuclidean, only that there is no such omnibus "space" in memory.

What is important for the geometry of mental representations is that it facilitates adaptive spatial behavior in the environment. The structure of ITM is not directly constrained by this need, though both its contents and any mapping procedures used to make this content explicit [9] are thus constrained. The environment may be expected to constrain the structure of WM representations. For instance, shepard [41] discusses environmental constraints on the form of internal representations that operate via evolutionary processes. He suggests that the geometric properties of the world of "perceiving, imagining, thinking, and dreaming" (WM representations) reflect the internalized constraints of a 3-dimensional and locally Euclidean world (except for a nonisotropy due to gravity). O'Keefe and Nadel [32] take a similarly Kantian-like position that the brain innately organizes experience in 3-dimensional, Euclidean terms because of environmental constraints.

To the degree that an attempt to establish the geometry of environmental knowledge is a cogent 
enterprise, it might be reformulated to refer to WM space rather than to LTM space or to some generic "mental" space. This is similar to one view of the recent analogue-propositional debate concerning the form of mental imagery ([20]; see [34] for a very different view of imagery): WM representations may very well function as if in an analogue, spatial medium even though the LTM representations from which they are generated may take any of several forms, including a propositional form. If one could establish that spatial estimation data reflected the simultaneous content of some WM space, one would be in a position to evaluate the geometric properties of the space.

As did Kosslyn, Ball, and Reiser [21], this endeavor asks what the metric of the spatial information preserved by visual images is. The time required to scan an image from location A to location $C$, for example, should never be greater than the time required to scan from $A$ to $B$ plus the time to scan from $B$ to $C$ (assuming equal scanning rates, etc.). Or one could examine angular estimates made from an image in order to determine if a Euclidean metric is a good description for it. Using such methods, one might even show that all WM spaces do not share a single geometry (e.g., even within the class of "large-scale" environments, variations in scale of size could be important).

An important and difficult question is whether one can actually determine which WM spaces are stored as internally consistent units in ITM and which are composed from a combination of two or more spatial units in LTM. In essence, what elements of the world (body zones, rooms, buildings, cities, etc.) are treated as single environmental units in various contexts? This is a difficult question to answer given that data reflecting the contents of LTM are expressed via WM (see [1] for a similar point about the analogue-propositional imagery debate). One could hold that metric consistency, equivalent scale and completeness within a set of spatial estimates is evidence of a unitary representation in LTM. But this would beg the question of mental geometry. It would never allow us to claim evidence against the metric nature of environmental knowledge (a conventionalist argument).

But the problem may not be intractable. Careful response-time studies might show that patterns of 
response-times in spatial estimation tasks vary as a function of whether: (a) a single environmental unit is being accessed across time, (b) moxe than one unit is being accessed sequentially across time, or (c) more than one unit is being accessed and simultaneously integrated because the particular estimation task requires coordinated access to multiple representations. It might also be informative to discuss metric inconsistencies with subjects (e.g., nonsymmetric distance estimates) after collecting a series of spatial estimates. Their responses to such queries might provide some insight into the cause of metric inconsistencies.

\section{Footnotes}

1 I am indebted to an anonymous reviewer for alerting me to these important caveats.

2 The relationship mediating $W M$ representations and behavior is also important; if significant transformations of the geometry of WM representations mediate this relationship, behavioral data will not directly inform us of WM geometry.

\section{References}

1. J.R.Anderson: Arguments concerning representations for mental imagery. Psychological Review 85, 249-277 (1978)

2. J.C.Baird: Psychophysical analysis of visual space. New York: Pergamon (1970)

3. J.C.Baird, M.Wagner, E.Noma: Impossible cognitive spaces. Geographical Analysis 14, 204-216 (1982)

4. A.A.Blank: Curvature of binocular visual space. Journal of the optical society of America 51, 335339 (1961)

5. B.P.Buttenfield: Comparing distortion on sketch maps and MDS configurationg. Professional Geographer 38 , $238-246(1986)$

6. M.T.Cadwallader: Cognitive distance in intraurban space. In: G.T.Moore, R.G.Golledge (eds.): 
Environmental knowing. Stroudsburg, PA: Dowden, Hutchinson \& Ross 1976, pp. 316-324

7. M.T.Cadwallader: Problems in cognitive distance: Implications for cognitive mapping. Environment and Behavior 11, 559-576 (1979)

8. D.Canter, S.Tagg: Distance estimation in cities. Environment and Behavior 7, 59-80 (1975)

9. R.M.Downs: Maps and mappings as metaphors for spatial representation. In: L.S.Liben, A.H.Patterson, N. Newcombe (eds.): Spatial representation and behavior across the life span. New York: Academic 1981, pp. 143-166

10. R.M.Downs, D.Stea: Cognitive maps and spatial behavior: Process and products. In: R.M.Downs, D.Stea (eds.): Image and environment. Chicago: Aldine 1973, pp. 8-26

11. J.M.Foley: The size-distance relation and the intrinsic geometry of visual space. Vision Research $12,323-332$ (1972)

12. T.Gärling, A.Böök, E.Lindberg, C.Arce: Evidence of a response-bias explanation of non-Euclidean cognitive maps. Professional Geographer 43, 143-149 (1991)

13. R.G.Golledge, L.J.Hubert: Some comments on nonEuclidean mental maps. Environment and Planning $A$ $14,107-118$ (1982)

14. A.Grünbaum: Philosophical problems of space and time, 2nd ed. Dordrecht, The Netherlands: D.Reidel (1973)

15. D.A.Hardwick, C.W.McIntyre, H.L.Pick: The content and manipulation of cognitive maps in children and adults. Monographs for the Society for Research in Child Development 41, 166 (1976)

16. K.J.Holyoak, W.A.Mah: Cognitive reference points in judgments of symbolic magnitude. Cognitive Psychology 14, 328-352 (1982) 
17. T.Indow: On geometry of frameless binocular perceptual space. Psychologia 17, 50-63 (1974)

18. W.H.Ittelson: Environment perception and contemporary perceptual theory. In: W.H.Ittelson (ed.): Environment and cognition. New York: Seminar 1973, pp. 1-19

19. P.R.Killeen: Geometric models of proximity data. Unpublished manuscript, Arizona state University, Tempe (1979)

20. S.M.Kosslyn: The medium and the message in mental imagery: A theory. Psychological Review 88, 46-66 (1981)

21. S.M.Kosslyn, T.M.Ball, B.J.Reiser: Visual images preserve metric spatial information: Evidence from studies of image scanning. Journal of Experimental Psychology: Human Perception and Performance 4, 4750 (1978)

22. B.Kuipers: The 'map in the head' metaphor. Environment and Behavior 14, 202-220 (1982)

23. B.Kuipers, T.S.Levitt: Navigation and mapping in large-scale space. Artificial Intelligence Magazine $73,25-43(1988)$

24. I.S.Iiben: Spatial representation and behavior: Multiple perspectives. In: L.S.Liben, A.H.Patterson, N. Newcombe (eds.): Spatial representation and behavior across the life span. New York: Academic 1981, pp. 3-36

25. R.Lloyd: Cognitive maps: Encoding and decoding information. Annals of the Association of American Geographers 79, 101-124 (1989)

26. R.K. Luneberg: The metric of binocular visual space. Journal of the Optical society of America 40, 637642 (1950)

27. K. Lynch: The image of the city. Cambridge, MA: 
M.I.T. Press (1960)

28. T.P.McNamara, J.K.Hardy, S.C.Hirtle: Subjective hierarchies in spatial memory. Journal of Experimental Psychology: Learning, Memory, and Cognition 15, 211-227 (1989)

29. I.Moar, G.H.Bower: Inconsistency in spatial knowledge. Memory and Cognition 11, 107-113 (1983)

30. D.R.Montello: The measurement of cognitive distance: Methods and construct validity. Journal of Environmental Psychology 11, 101-122 (1991)

31. J.Muller: Non-Euclidean geographic spaces: Mapping functional distances. Geographical Analysis 14, 189203 (1982)

32. J.O'Keefe, L.Nadel: The hippocampus as a cognitive map. New York: Oxford University Press (1978)

33. J.Piaget, B.Inhelder: The child's conception of space. London: Routledge \& Kegan Paul Ltd. (1956) (Original work published 1948)

34. Z.W.Pylyshyn: The imagery debate: Analogue media versus tacit knowledge. Psychological Review 88, 1645 (1981)

35. J.J.Rieser, J.L.Lockman, H.L.Pick: The role of visual experience in knowledge of spatial layout. Perception and Psychophysics 28, 185-190 (1980)

36. E.K.Sadalla, W.J.Burroughs, L.J.Staplin: Reference points in spatial cognition. Journal of Experimental Psychology: Human Learning and Memory 6, 516-528 (1980)

37. W.C.Salmon: space, time, and motion: A philosophical introduction. Dickenson (1975)

38. M.Schlick: The four-dimensional world. In: J.J.C.Smart (ed.): problems of space and time. New York: The Macmillan Company 1964, pp. 292-296 (Reprinted from chap. 7 of The philosophy of nature, 
translated by A.von Zeppelin, Philosophical Library, New York, 1949)

39. R.N.Shepard: Attention and the metric structure of the stimulus space. Journal of Mathematical Psychology 1, 54-87 (1964)

40. R.N.Shepard: Representation of structure in similarity data - problems and prospects. Psychometrika 39, 373-422 (1974)

41. R.N.Shepard: Ecological constraints on internal representation: Resonant kinematics of perceiving, imagining, thinking, and dreaming. Psychological Review 91, 417-447 (1984)

42. I.Sklar: space, time, and spacetime. Berkeley: University of California (1974)

43. D.Stea: The measurement of mental maps: An experimental model for studying conceptual spaces. In: K.R.Cox, R.G.Golledge (eds.): Behavioral problems in geography: A symposium. Evanston, IL: Northwestern University 1969 , pp. 228-253

44. P.W.Thorndyke, B.Hayeg-Roth: Differences in spatial knowledge acquired from maps and navigation. Cognitive Psychology 14, 560-581 (1982)

45. W.Tobler: The geometry of mental maps. In: R.G.Golledge, G.Rushton (eds.): Spatial choice and spatial behavior. Columbus: Ohio State University 1976, pp. 69-81

46. A.Tversky: Features of similarity. Psychological Review 84, 327-352 (1977) 